\title{
Imagen del drogodependiente y el enfermo mental entre los profesionales de la salud
}

\author{
Braña, B.*; Rodríguez Díaz, F. J.*; Cuesta, M.*; Bobes, J.**; Gómez, P.*; Sálz, P.A.* \\ (*) Grupo de Investigación Psicosocial. Facultad de Psicología. Universidad de Oviedo \\ $\left({ }^{*}\right)$ Área de Psiquiatría. Facultad de medicina. Universidad de Oviedo \\ Enviar correspondencia a: \\ F.J. Rodríquez Díaz. Universidad de Oviedo. Facultad de Psicología. Plaza de Feijoo s/n; 33003 OVIEDO. Tel.: 985 10 32 55/ E-Mail: gallego@sci.cpd.uniovi.es
}

\section{RESUMEN:}

El objetivo de este trabajo es realizar un análisis comparativo de la imagen social de los drogodependientes y enfermos mentales. Para ello planteamos, a una muestra de 152 profesionales de la Salud Mental, diversas cuestiones relacionadas con el conocimiento y la interacción con drogodependientes y enfermos mentales: personas con trastorno psicótico, trastorno de la personalidad, trastorno adaptativo y trastorno neurótico. Nuestros resultados indican que la mitad de la muestra considera al drogodependiente un enfermo mental y ubica al adicto en una posición intermedia entre los trastornos psicóticos graves y los trastornos adaptativos y neuróticos.

Palabras Clave: Drogodependencia / Enfermedad mental / Imagen social / Profesionales de salud mental

\begin{abstract}
:
The goal of this research is carry out a comparative analysis of the social image of the drug dependant and mental illness. A sample of 152 Mental Health workers were questioned on their knowledge and opinion of drug dependants and those who suffer from a mental illness: psychotic disorder, personality disorder, adaptive disorder and neurotic disorder. The results indicate that half the sample considers that drug dependency to be a mental illness and situates the addict in a position midway between serious psychotic disorders and adaptive and neurotic disorders.
\end{abstract}

Key words: drug dependency, mental illness, social image, mental health professionals

\section{INTRODUCCIÓN}

$\mathbf{L}$ a imagen que la sociedad tiene del adicto a drogas se articula en torno a informaciones, ideas y escenas que habitualmente reflejan comportamientos alejados de lo socialmente normativo: marginalidad y desviación social. Pero también incorpora elementos de enfermedad o vicio, consideraciones morales y legales. En definitiva, las opiniones sobre las drogas y quienes las consumen están mediatizadas por el sustrato de valores, creencias y actitudes de la sociedad.

Se plantea, pues, la necesidad de reflexionar sobre la sociedad que genera el estereotipo del drogodependiente, que etiqueta características y comportamientos como desviados, indeseables o marginales y que crea una percepción de amenaza para después analizarla bien como un problema de legalidad, como una enfermedad o como un problema moral. Los medios de comunicación de masas como ineludibles transmisores de información, aún hoy en día, suelen identificar droga con ilegalidad, lo que ratifica la conceptualización del adicto (sobre todo del consumidor de heroína) como un desviado, colocando en un plano radicalmente diferente a otras sustancias "legales" con potencial altamente adictivo de mayor consumo en nuestra sociedad: alcohol, tabaco, psicofármacos... etc. (Pinazo, 1993; Froján, Rubio y Peris, 1994).

El estigma de lo desviado tampoco es ajeno a las personas que padecen una enfermedad mental. El diagnóstico psiquiátrico supone una marca que aleja a quién lo presenta de la normalidad, atribuyéndole menor responsabilidad, mayor peligrosidad... (Steadman, Mulvey, Monahan y otros., 1998).

En el devenir de la historia nos encontramos alusiones al consumo de sustancias que guardan cierto paralelismo con las interpretaciones vigentes en otras épocas sobre la enfermedad mental. Así, Fernández 
D’Oviedo y Valdez transmite en sus crónicas del Nuevo Mundo como los indígenas de la Española utilizaban como medio de ponerse en contacto con los dioses rituales en los que el consumo de drogas o pócimas era un elemento central, con ilustrativos pasajes "el espíritu contestaba de modo exacto por la boca del hombre (hechicero que se había tomado una pócima), que se hallaba en estado de éxtasis" (Inglis, 1994, pág. 12). El propio cronista relata como el proceso acometido por el hechicero, las convulsiones y la emisión de voces extrañas le recuerdan un fenómeno nada extraño a la Europa postmedieval: la posesión diabólica. Así pues, parece que aún cuando el fenómeno del consumo de sustancias estaba contextualizado en un medio en que no constituía un problema social y cuando no se hablaba de fenómenos como la dependencia se alude por la similitud sintomática a una explicación manejada para dar cuenta de los comportamientos extraños e inexplicables: posesión diabólica. La vinculación de enfermedad mental, pócimas (drogas) e influencia demoniaca la encontramos en no pocos casos de víctimas de la hoguera (Foucault, 1991; González Duro, 1994).

La imagen social del drogodependiente y del enfermo mental, por tanto es posible sostener que conectan en varios puntos: rechazo, marginalidad, conductas violentas. Incluso su tratamiento médico/psicológico se realiza desde similares dispositivos de salud: comunidades terapeúticas, centros de día... Tampoco son ajenos al sentimiento social los objetivos de los programas de tratamiento de personas con adicción a sustancias o enfermedad mental y que se expresan en términos como: contención en crisis, atención normalizada, reintegración / reinserción social.

La integración normalizada de la persona estigmatizada igualmente se plantea incrementando la comprensión objetiva del fenómeno en su justa realidad contra la mitificación, el tremendismo y los estereotipos vigentes, evitando actitudes y reacciones sociales de dramatización, miedo y rechazo agresivo sobre los drogodependientes y alentando actitudes positivas de concienciación, prevención y apoyo a la recuperación e inserción social del drogodependiente. Lo que redunda en el incremento de la información - formación de la opinión pública, colectivos sociales y profesionales y medios de comunicación.

La comorbilidad adicción a sustancias - alteración psicopatológica es un aspecto que puede ejercer determinada influencia en la forma de percibir en la actualidad al drogodependiente desde el momento en que, sin entrar en consideraciones causales, incrementa el parecido entre ambos colectivos.
Lo anteriormente expuesto nos ha llevado a plantearnos: ¿qué sustrato de opinión subyace en los profesionales de la salud (como expertos en la valoración y tratamiento de estas patologías) tanto con relación a los trastornos por adicción como a otras alteraciones psiquiátricas?. Es decir, ¿Se mantienen el estigma de la cronicidad / incurabilidad?, ¿Qué agente consideran como causante de estos fenómenos?, ¿Es el drogodependiente / enfermo mental capaz de llevar una vida normalizada, en los ámbitos sociales: personal, laboral? ... De esta manera, el objetivo de este trabajo es realizar una comparación de las opiniones de profesionales de la salud mental sobre el drogodependiente con las opiniones sobre otros trastornos mentales (Esquizofrenia y otros Trastornos psicóticos, Trastornos de la personalidad, Trastornos adaptativos y Trastornos neuróticos), concretado en los siguientes puntos:

- Valorar la existencia de la imagen del drogodependiente como enfermo mental.

- Comprobar la opinión de los profesionales sobre los trastornos adictivos en comparación con otros trastornos mentales.

- Analizar la existencia de diferencias entre categorías de profesionales.

\section{MATERIAL Y MÉTODOS}

La muestra utilizada $(\mathrm{N}=152)$ ha sido conformada por profesionales de la salud que han contestado (de forma anónima y voluntaria) a una encuesta ad hoc enviada a todos aquellos que se encontraban vinculados con Salud Mental (Red Pública y Privada) de la Comunidad Autónoma del Principado de Asturias, entre los meses de Marzo y Junio de 1998.

Los datos se recogieron a través de las respuestas a items alusivos a la percepción del drogodependiente y del enfermo mental (Anexo I). Los items fueron valorados en una escala tipo Likert de cinco opciones de respuesta, en la que se pedía la opinión de los encuestados con relación a las cinco siguientes alteraciones: Trastornos adictivos, esquizofrenia y trastornos psicóticos, trastornos de la personalidad, trastornos de adaptación y trastornos neuróticos.

EL $76,6 \%$ de la muestra son mujeres y el $23,4 \%$ hombres, con una media de edad de 35,8 años y una desviación típica de 6,8 años en un rango que oscila desde los 22 hasta los 62 años. La experiencia laboral en salud mental es de 9,54 años como media con desviación típica de 7,13 años, en un rango que oscila 


\begin{tabular}{|c|c|c|c|c|c|}
\hline ITEMS & \multicolumn{5}{|c|}{ RESPUESTAS } \\
\hline $\begin{array}{l}\text { 1. Señale qué factor influye más en la etiolc } \\
\text { enfermedades mentales: } \\
\text { 1a) Esquizofrenia y otros trastornos } \\
\text { 1b) Trastornos de la personalidad } \\
\text { 1c) Trastornos adictivos } \\
\text { 1d) Trastornos adaptativos } \\
\text { 1e) Trastornos neuróticos }\end{array}$ & $\begin{array}{l}\text { oria de } 1 \text { qas } \\
\text { psicóticds } \\
\text { Sociedađ }\end{array}$ & $\begin{array}{l}\text { s siguien } \\
\text { Familia }\end{array}$ & Individu & 1oHerencip & $\begin{array}{l}\text { Lo } \\
\text { desconoz }\end{array}$ \\
\hline $\begin{array}{l}\text { 2. ¿Qué porcentaje de curación tienen las si } \\
\text { enfermedades mentales?: } \\
\text { 2a) Esquizofrenia y otros trastornos } \\
\text { 2b) Trastornos de la personalidad } \\
\text { 2c) Trastornos adictivos } \\
\text { 2d) Trastornos adaptativos } \\
\text { 2e) Trastornos neuróticos }\end{array}$ & $\begin{array}{l}\text { guientes } \\
\text { psicóticos } \\
\text { Muy altф }\end{array}$ & Al to & Medio & Bajo & Muy baj \\
\hline $\begin{array}{l}\text { 3. Los pacientes mentales son capaces de rea } \\
\text { cualificadas: } \\
\text { 3a) Esquizofrenia y otros trastornos } \\
\text { 3b) Trastornos de la personalidad } \\
\text { 3c) Trastornos adictivos } \\
\text { 3d) Trastornos adaptativos } \\
\text { 3e) Trastornos neuróticos }\end{array}$ & $\begin{array}{l}\text { lizar labs } \\
\text { psicóticos } \\
\text { Muy de } \\
\text { acuerdo }\end{array}$ & $\begin{array}{c}\text { De } \\
\text { acuerdo }\end{array}$ & Indecis & 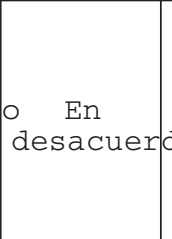 & $\begin{array}{c}\text { Muy en } \\
\text { dowesacuer }\end{array}$ \\
\hline $\begin{array}{l}\text { 4. ¿Qué pacientes mentales deberían ser privad } \\
\text { 4a) Esquizofrenia y otros trastornos } \\
\text { 4b) Trastornos de la personalidad } \\
\text { 4c) Trastornos adictivos } \\
\text { 4d) Trastornos adaptativos } \\
\text { 4e) Trastornos neuróticos }\end{array}$ & $\begin{array}{l}\text { ps de tene } \\
\text { psicóticos } \\
\text { Muy de } \\
\text { acuerdo }\end{array}$ & $\begin{array}{c}\text { r hijos?: } \\
\text { De } \\
\text { acuerdo }\end{array}$ & Indecis & O En & $\begin{array}{c}\text { Muy en } \\
\text { dodesacuer }\end{array}$ \\
\hline 5. El drogodependiente es un enfermo mental & $\begin{array}{l}\text { Muy de } \\
\text { acuerdo }\end{array}$ & $\begin{array}{c}\text { De } \\
\text { acuerdo }\end{array}$ & Indecis & O En & $\begin{array}{r}\text { Muy en } \\
\text { dodesacuer }\end{array}$ \\
\hline
\end{tabular}

entre los 42 años de trabajo en salud mental y menos de un año. La mayor parte de la muestra lleva trabajando o bien menos de 5 años o de 5 a 10 años con pacientes mentales.

La mayoría de los profesionales encuestados trabajan en los Servicios de Salud del Principado de Asturias $(72 \%)$ siendo las profesiones más representadas ATS / DUE (29\%) y Psicólogos (28,6\%) (Tabla I).

Como apoyo metodológico para valorar la significatividad de las comparaciones entre las variables sociodemográficas (sexo, categoría profesional y años de experiencia) se han utilizado la prueba $\mathrm{K}$ de Kruskall Wallis para $\mathrm{K}$ muestras independientes y la prueba $\mathrm{U}$ de Mann Whitney para dos muestras independientes, según el caso. Así mismo hemos realizado un análisis de medidas repetidas para la comparación de significatividad de las respuestas entre los trastornos adictivos y las restantes categorías de trastornos mentales. EI paquete estadístico utilizado fue el SPSS versión 9.0.

\section{RESULTADOS}

El $47 \%$ de la muestra atribuye a los trastornos adictivos causalidad social, mientras que el $40 \%$ de la muestra opina que la causa es fundamentalmente individual, mientras una cuarta parte de los profesionales también considera importante la influencia de la familia. 1). Los profesionales diferencian fundamentalmente (Tabla II.1) la etiología de los trastornos adictivos en relación con los trastornos psicóticos y esquizofrenia básicamente atribuídos a causas hereditarias.

El porcentaje de curación de los trastornos adictivos es medio para el $44,4 \%$ y bajo para el $31,1 \%$ de la muestra, hallándose diferencias con significación estadística $(K<$.005) con la estimación de curación de los trastornos psicóticos y esquizofrenia, que se considera muy baja por el $53,7 \%$ de la muestra; con los trastornos de personalidad en las estimaciones media y muy baja y con los trastornos adaptativos y "neuróti-

1) En el item I (Anexo I) si bién en el cuestionario se especificaba elegir una opción en relación con la etiología, una elevada proporción de encuestados señalaron dos o más opciones, de ahí que la suma de los porcentajes de respuestas supere el lógico 100\%. Este es el motivo por el que el análisis del item ha sido únicamente descriptivo. 
Tabla 1. Distribución de variables sociodemográficas en la muestra

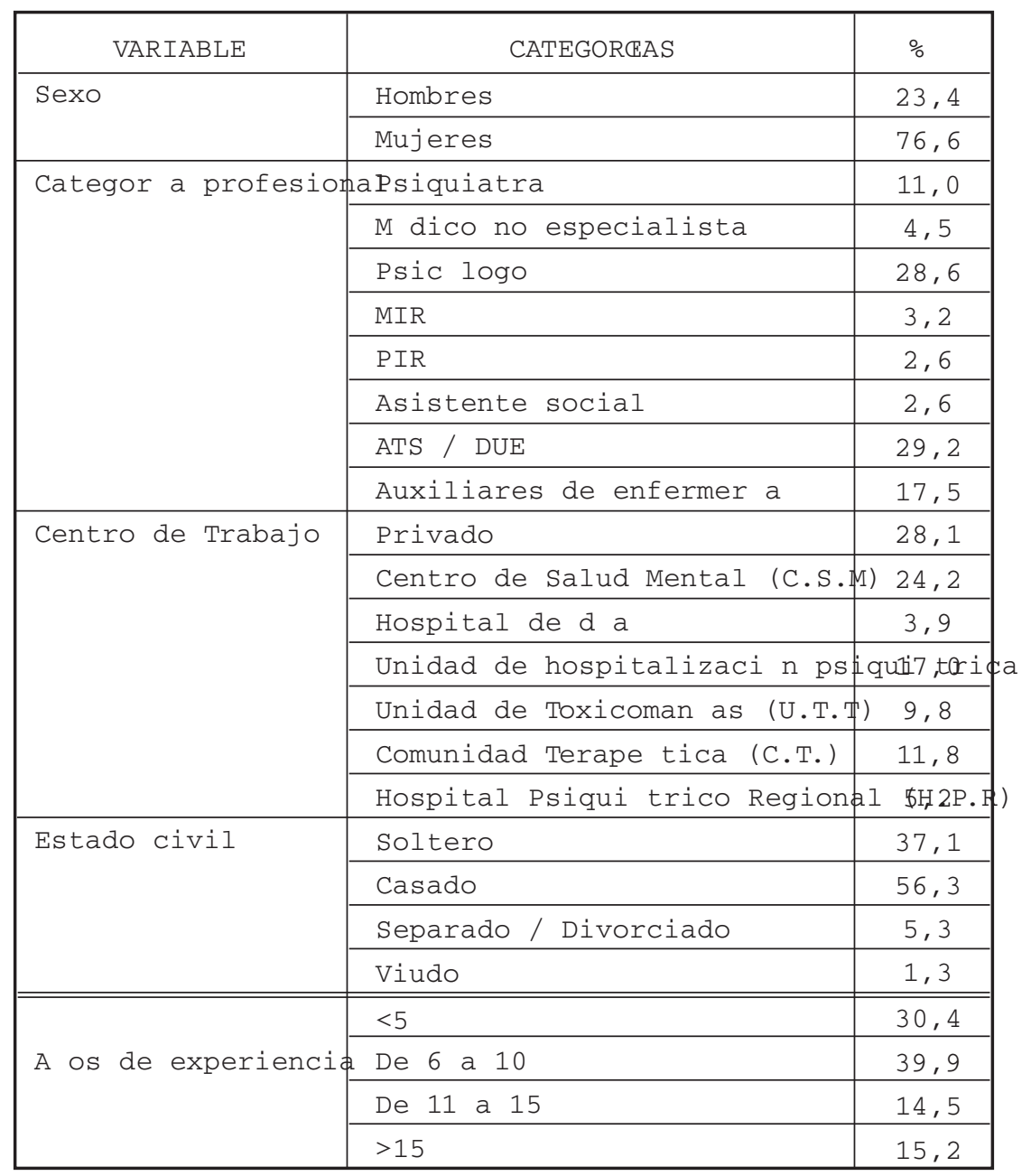

cos" aquienes se atribuyen estimaciones de curación más positivas.

Respecto a las variables sociodemográficas (Tabla III) hemos encontrado diferencias con significación estadística en la comparación entre categorías profesionales, observándose que, en general los profesionales de enfermería y el personal auxiliar opinan que el porcentaje de curación es bajo o muy bajo.

La mayoría de los profesionales de salud mental $(63,6 \%)$ consideran capaz a la persona con trastorno adictivo de realizar labores profesionales que requieren cualificación. Cuando comparamos el trastorno adictivo con las restantes patologías encontramos que la muestra considera más capaces para desenvolverse adecuadamente en el ámbito laboral a las personas con patología adaptativa o neurótica que al drogodependiente y a este más capaz que a las personas con esquizofrenia o trastorno psicótico (Tabla II.3). Aquí, si embargo, no hemos encontrado diferencias con signi- ficación estadística respecto a la categoría profesional, sexo o los años de experiencia.

La mayoría de los profesionales $(49,3 \%$ en desacuerdo / muy en desacuerdo) no considera que se deba privar a la persona que padece trastorno adictivo de tener hijos, aún cuando la tendencia normalizadora se mantiene en el resto de las patologías psiquiátricas, las pruebas de significación estadística presentan diferencias en las respuestas ante la esquizofrenia y trastornos psicóticos (un porcentaje superior de profesionales considera que han de ser privados de tener hijos) y con los Trastornos adaptativos y "neuróticos" ante los cuales los profesionales son menos restrictivos (Tabla II.4).

Las comparaciones en función de la categoría profesional vuelven a ofrecer significación estadística, observándose una tendencia estigmatizante más elevada en profesionales de enfermería (auxiliares de enfermería y ATS). 
Tabla II Resultados análisis de medidas repetidas (* Comparaciones con significación estadística al .05)

\begin{tabular}{|c|c|c|c|c|c|c|}
\hline \multicolumn{2}{|r|}{ Item1 } & SOCIEDAD & FAMILIA & NDIVIDUO & HERENCIA & $\mathrm{NS} / \mathrm{NC}$ \\
\hline 1.a) & Esquizofrenia y otros transto & rnoss, eps: & CÓ\&்̇,CQS & 31,1 & 50,3 & 6,6 \\
\hline 1.b) & Transtornos de la Personalidad & 11,3 & 42,0 & 44,0 & 10,0 & 6,7 \\
\hline 1.C) & Transtornos adictivos & 47,0 & 24,5 & 40,4 & 1,3 & 4,0 \\
\hline 1.d) & Transtornos adaptativos & 39,3 & 37,3 & 30,7 & 2,7 & 7,3 \\
\hline 1.e) & Transtornos neuróticos & 5,3 & 35,8 & 57,0 & 6,6 & 6,0 \\
\hline
\end{tabular}

Tabla II.1. Etiología (Señale que factor influye más en la etiología de las siguientes enfermedades mentales)

\begin{tabular}{|c|c|c|c|c|c|c|}
\hline & Item 2 & $\underset{\frac{\%}{\%}}{\text { Muy }}$ alt & $\begin{array}{l}\text { Al to } \\
\%\end{array}$ & $\begin{array}{l}\text { Medio } \\
\%\end{array}$ & $\begin{array}{c}\text { Bajo } \\
\%\end{array}$ & $\underset{\%}{\text { Muy Bajd }}$ \\
\hline $2 . a)$ & Esquizofrenia y otros transto & rnos ps & ic $\mathrm{C} t$, iAcos & $8,1 *$ & 34,9 & $53,7 *$ \\
\hline $2 . \mathrm{b})$ & Transtornos de la Personalidad & 0,7 & 6,7 & $28,2 *$ & 34,2 & $30,2 *$ \\
\hline $2 . c)$ & Transtornos adictivos & 2,0 & 11,9 & 44,4 & 31,1 & 10,6 \\
\hline $2 . d)$ & Transtornos adaptativos & $20,1 *$ & $39,6 *$ & $31,5 *$ & 7,4 * & 1,3 * \\
\hline $2 . e)$ & Transtornos neuróticos & 14,6 * & 29,8 * & $30,5 *$ & 19,2 * & 6,0 \\
\hline
\end{tabular}

Tabla II.2. Porcentaje de curación. (¿Qué porcentaje de curación tiene las siguientes enfermedades mentales?)

\begin{tabular}{|c|c|c|c|c|c|c|}
\hline & Item 3 & $\begin{array}{l}\text { Muy de } \\
\text { acuerdo } \\
\%\end{array}$ & $\begin{array}{c}\text { De acuer } \\
\frac{\%}{\sigma}\end{array}$ & $\begin{array}{c}\text { doIndecis } \\
\%\end{array}$ & $\begin{array}{c}\text { En } \\
\text { odesacuer } \\
\% \\
\%\end{array}$ & $\begin{array}{c}\text { Muy en } \\
\text { dzes a cuer } \\
\%\end{array}$ \\
\hline 3.a) & Esquizofrenia y otros transt & prn8: 0ps & 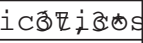 & 20,0 & $29,3 *$ & 5,3 \\
\hline $3 . \mathrm{b})$ & Transtornos de la Personalida & d 9,5 & 57,4 & 18,2 & 14,2 & 0,7 \\
\hline 3.c) & Transtornos adictivos & 9,5 & 54,1 & 16,9 & 18,2 & 1,4 \\
\hline $3 . d)$ & Transtornos adaptativos & $15,8 *$ & 61,0 & 11,0 & 11,0 & 0,7 \\
\hline $3 . e)$ & Transtornos neuróticos & $21,5 *$ & 59,1 & 9,4 * & 9,4 * & 0,7 \\
\hline
\end{tabular}

Tabla II.3. Restricciones laborales (Los pacientes mentales son capaces de realizar labores cualificadas.)

\begin{tabular}{|c|c|c|c|c|c|}
\hline Item 4 & $\begin{array}{c}\text { Muy de } \\
\text { acuerdo } \\
\%\end{array}$ & De acuer & $\begin{array}{c}\text { doIndecis } \\
\%\end{array}$ & $\begin{array}{c}\text { En } \\
\text { spatesacuer } \\
\%\end{array}$ & $\begin{array}{c}\text { Muy en } \\
\text { dwes acuer } \\
\%\end{array}$ \\
\hline 4.a) Esquizofrenia y otros transto & rnds, 2 s & 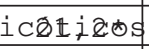 & 21,2 & $22,6 *$ & 26,7 \\
\hline 4.b) Transtornos de la Personalidad & d 1,5 & 10,2 & 22,6 & 35,0 & 30,7 \\
\hline 4.c) Transtornos adictivos & 2,2 & 10,9 & 20,4 & 37,2 & 29,2 \\
\hline 4.d) Transtornos adaptativos & - & $2,9 *$ & 18,2 & 46,0 * & $32,8 *$ \\
\hline 4.e) Transtornos neuróticos & 2,1 & $2,1 *$ & 17,9 & $42,9 *$ & $35,0 *$ \\
\hline
\end{tabular}

Tabla II.4. Restricciones personales (¿Qué pacientes mentales deberán ser privados de tener hijos?) 
Tabla III. Items con significación estadística en la variable Categoría profesional.

\begin{tabular}{|c|c|c|c|c|}
\hline \multicolumn{2}{|r|}{ Items } & Sexo & $\begin{array}{c}\text { Experiencia } \\
\text { laboral }\end{array}$ & $\begin{array}{l}\text { Categoría } \\
\text { profesional }\end{array}$ \\
\hline $2 . a)$ & Esquizofrenia y otros transth & prnos Ü. iø & n.s. & $\mathrm{K}=.000$ \\
\hline $2 . b)$ & Transtornos de la Personalida & d n.s. & n.s. & $K=.000$ \\
\hline $2 . c)$ & Transtornos adictivos & n.s. & $K=.026$ & $K=.030$ \\
\hline $2 . d)$ & Transtornos adaptativos & n.s. & n.s. & $\mathrm{K}=.000$ \\
\hline $2 . e)$ & Transtornos neuróticos & \begin{tabular}{|c|}
$\mathrm{n} . \mathrm{S}$. \\
\end{tabular} & n.s. & $\mathrm{K}=.000$ \\
\hline 4.a) & Esquizofrenia y otros transt & prnos pisisóticoß & n.s. & $K=.000$ \\
\hline $4 . \mathrm{b})$ & Transtornos de la Personalida & a $\quad \mathrm{n} . \mathrm{s} . \mathrm{P}$ & n.s. & $K=.002$ \\
\hline $4 . \mathrm{C})$ & Transtornos adictivos & n.s. & n.s. & $\mathrm{K}=.000$ \\
\hline $4 . d)$ & Transtornos adaptativos & n.s. & n.s. & $K=.004$ \\
\hline $4 . e)$ & Transtornos neuróticos & $\mathrm{n} . \mathrm{s}$. & $\mathrm{n} . \mathrm{s}$. & $K=.004$ \\
\hline
\end{tabular}

Por último, señalar que en torno al $50 \%$ de la muestra considera que el drogodependiente es un enfermo mental, tal y como se plantea en el enunciado, mientras que no están de acuerdo con dicha afirmación el $34 \%$ de los encuestados. Aquí nuevamente no hemos observado diferencias con significación estadística en ninguna de las variables sociodemográficas contempladas.

\section{DISCUSIÓN}

Nuestros resultados, al igual que los obtenidos por Rabkin (1972) y Trute, Tefft y Segal (1989), indican la existencia de una imagen social negativa hacia el drogodependiente y enfermo mental, que se sustenta entre otros elementos en la creencia de incurabilidad teniendo en cuenta la idea de cronicidad resulta comprensible la diferencia existente en las respuestas de los profesionales de la muestra entre alteraciones percibidas como más o menos graves -. El trastorno crónico se entiende que conlleva importantes costes sociales que hacen necesario un modelo de abordaje y tratamiento ajustado a sus necesidades especiales y que supone un importante esfuerzo a nivel de entrenamiento de profesionales, prevención y tratamiento (Lucas, Loutsch y Frances, 1985). De esta manera, la categoría profesional, así como los años de experiencia laboral con enfermos mentales y drogodependientes influye en la actitud ante estos pacientes, hecho que también ha comprobado en sus resultados Carroll (1996).

La (re)- integración laboral de la persona con trastorno adictivo es una cuestión fundamental para poder hablar de integración social normalizada, como ya ha constatado Ladewig (1993). Un desempeño ocupacional adecuado no es incompatible con la adicción a sustancias, como también refiere Nelson (1981) al observar que numerosos adictos a varias sustancias desempeñan de forma adecuada su trabajo al tiempo que estaban realizando programas de tratamiento. Ello, a su vez, no excluye que una elevada proporción de drogodependientes no desempeñen una ocupación productiva o bien lo hagan de forma marginal. Tampoco es fácil la reintegración de las personas con trastorno psicótico grave al medio laboral lo que viene a confirmar que a mayor grado de estigmatización menores son las posibilidades de normalización (Grossi, Rodríguez, Cuesta y otros, 1999).

La discusión sobre la capacidad de los adictos a drogas, pero también de las personas con enfermedad mental grave, para cuidar adecuadamente de sus hijos no es un tema ajeno a nuestro medio. La bibliografía refiere problemas pre- y peri- natales, de crianza así como la mayor frecuencia de hijos que crecen fuera del núcleo familiar (Mark, 1983; Conte, Mazzoni, Serretti y otros, 1994 y Hawley, Halle, Drasin y Thomas, 1995) sobre todo en madres adictas a sustancias, fundamentalmente heroína y cocaína; al contrario, autores como Van Baar (1990) no encuentran diferencias en el desarrollo de hijos de madres con problemas de drogodependencia.

La conceptualización del drogodependiente como enfermo mental es deudora de la aceptación de un modelo médico de enfermedad, que como hemos observado coexiste tanto en el ámbito social como en la práctica profesional con otras teorías explicativas (Furnham, 1996) que dan a la adicción a sustancias un perfil pluridimensional. Los resultados parecen cohe- 
rentes con los obtenidos por Acker (1993), que revisando los modelos explicativos de la conducta adictiva vigentes en la actualidad concluye que el modelo prevalente de adicción incorpora elementos sociales y comportamentales.

En definitiva, de los que se trata es de modificar la cognición social o representación social. Los individuos tienden a confirmar sus propias expectativas (profecía autocumplida), pero resulta del todo necesario el desconfirmar dicha forma de razonamiento. Es posible, aunque la tarea no es nada fácil. Sin embargo sí se han obtenido resultados positivos. Éstos, a su vez, son tanto mejores cuanta más información tengan los individuos acerca de los orígenes, estructura y dinámcia de la enfermedad mental (Morrison, 1976, 1977; Morrison, Cocozza y Vanderwyst, 1980; Morrison y Teta, 1980; Quicke, Beasley y Morrison, 1990; Smith, 1990; Corrigan y Penn, 1997, 1999; Corrigan, 1998; Corrigan, River, Lundin, Wasowski, Campion, Mathisen, Goldstein, Gagnon, Bergman, y Kubiak, 1999; Heresco-Levy, Ermilov, Giltsinsky, Lichtenstein y Blander, 1999; Homes, Corrigan, Williams, Canar y Kubiak, 1999; Wahl, 1999).

\section{CONCLUSIONES}

1. Los profesionales de salud mental de la muestra mantienen la imagen del drogodependiente como enfermo mental.

2. Aún cuando los profesionales consideran al trastorno adictivo una enfermedad mental, la imagen de este difiere fundamentalmente de la representación que los profesionales tienen de los trastornos psicóticos por una parte y de los trastornos adaptativos y "neuróticos" por otra. De esta manera, las personas con esquizofrenia o trastorno psicótico son percibidos como más graves, con etiología en menor medida modificable y con menores posibilidades de reintegración laboral y personal. Las personas con trastorno adictivo son ubicadas en un estatus intermedio, conservando una imagen social más deteriorada que otros pacientes con alteraciones de índole neurótica o adaptativa.

3. La imagen social del adicto, así como de los restantes trastornos contemplados en el presente estudio varía según la categoría profesional de los encuestados, no así en función del sexo y los años de experiencia laboral.

\section{AGRADECIMIENTOS}

Este trabajo se enmarca en un proyecto dirigido a analizar las actitudes y representaciones sociales de la enfermedad mental en personas relacionadas por su formación académica o profesional con la enfermedad mental financiado por la Fundación para el Fomento en Asturias de la Investigación Científica Aplicada y la Tecnología. ((FICYT) con referencia PB-PGI97-06.

El proyecto ha contado, así mismo, con la conformidad de los Servicios de Salud del Principado de Asturias (SESPA), a quienes agradecemos las facilidades que nos han dado para acceder a los dispositivos de la red de Salud Mental.

\section{BIBLIOGRAFÍA}

Carroll, J. (1996). Attitudes to drug users according to age of staff. Prof. Nurse. Mar; 11(6) 401-4.

Conte, G.L.; Mazzoni, S.; Serretti, A.; Fundaro, C. y Tempesta, E. (1994). Acta Paediatrica Suppl.; 404: 47-53.

Corrigan, P.W. (1998). The impact of stigma on severe mental illness. Cognitive and Behavioral Practice, 5, 201-222.

Corrigan, P.W. y Penn, D.L. (1997). Disease and discrimination: Two paradigms that describe severe mental illness. Journal of Mental Health, 6, 355-366.

Corrigan, P.W. y Penn, D.L. (1999). Lessons from social psychology on discrediting psychiatry stigma. American Psychologist, 54, 765-776.

Corrigan, P.W., River, L.P., Lundin, R.K., Wasowski, K.U., Campion, J., Mathisen, J., Goldstein, H., Gagnon, C., Bergman, M.y Kubiak, M.A. (1999). Predictors of participation in campigns against mental illness stigma. Journal of Nervous and Mental Disease, 187, 378-380.

Foucault, M. (1991) Historia de la locura en la época clásica (2 Vol.). Madrid: Fondo de Cultura económica.

Froján, M.X.; Rubio,R. Y Peris, B. (1994). La imagen de la droga en la prensa española. Revista española de drogodependencias, 19(3), 191-204.

Furnham, A. Thomson, L. (1996). Lay theories of heroin addiction. Social Science Medicine, Jul; 43(1): 29-40.

González Duro, E. (1994). La Historia de la locura en España (3. Vol.) Madrid: Temas de hoy.

Grossi, F.J.; Rodríguez, F.J.; Cuesta, M. y otros (1999). Actitudes hacia la enfermedad mental en profesionales y futuros profesionales de la Salud Mental. Sesgos y desviaciones. Oviedo: Informe de Investigación no publicado para FICYT. 
Hawley, T.L.; Halle, T.G.; Drasin, R.E. y Thomas, N.G. (1995). Children of addicted mothers: effects of the "crack epidemic" on the caregiving enviroment and the development of preschoolers. American Journal of Orthopsychiatry. Jul; 65(3): 364-79.

Heresco-Levy, U., Ermilov, M., Giltsinsky, B., Lichtenstein, M. y Blander, D. (1999). Treatment-resistant schiophrenia and staff rejection. Schizophrenia Bulletin, 25, 457-465.

Inglis, B. (1994). El juego prohibido. La Historia Social de las drogas. Gerona: Tikal.

Ladewig, D. (1993). The importance of work and unemployement for the long - term outcome and rehabilitation of drug dependent patients. The Umsh, Mar; 50(3): 189193.

Lucas, J.J.; Loustch, E, y Frances, R.J. (1985). The drug addict as chronic patient. Psychiatryc Medicine; 3(4): 427-444.

Llopis, D.; Pons, J. y Berjano, E. (1996). Evaluación diferencial de la imagen social de los consumidores de drogas. Psicothema, Vol. 8, n³: 465-474.

Morrison, J.K. (1976). Demythologizing mental patient's attitudes toward mental illness: An empirical study. Journal of Community Psychology, 4, 181-185.

Morrison, J.K. (1977). Changing negative attributions to mental patients by means of demythologizind seminars. Journal of Clinical Psychology, 4, 549-551.

Morrison, J.K., Cocozza, J.J. y Vanderwyst, D. (1980). Anattempt to cahnge the negative, stigmatizing image of mental patients through brief reeducation. Psychological Reports, 47, 334.

Morrison, J.K. y Teta, D.C. (1980). Reducing students fear of mental illness by means of seminar-induced belief change. Journal of Clinical Psychology, 36, 275-276.
Nelson, J.E. (1981). Drug abusers on the job. Journal of occupational Medicine, June; 23(6): 403-408.

Pinazo, S. (1993). Programa de prevención del consumo de drogas en niños en edad escolar dirigido a padres. Universidad de Valencia: Tesis doctoral no publicada.

Quicke, J., Beasley, K. y Morrison, C. (1990). Challenging prejudice through education: The story of a mental handicap awaresness curriculum project. Bristos, Falmer Press.

Rabkin, J.G. (1972).- Opinions about mental illness: A review of the literature. Psychological Bulletin, Vol.77, $N^{\circ} 3$; 153-171

Rodríguez Cabrera, J. (1988). Drogodependencias: Un enfoque multidisciplinario. Madrid: Plan Nacional sobre Droga.

Smith, A. (1990). Social influence and antiprejudice training programs. En J. Edwards, R.S. Tindale, L. Heath y E.J. Posavac (Eds.), Social influence processes and prevention (pp. 183-196). New York, Plenum Press.

Steadman, H.J.; Mulvey, E.P.; Monahan, J.; Robbins, P.C.; Appelbaum, P.S.; Grisso, T.; Roth, L.H. y Silver, E. (1998). Violence by people discharged from acute psychiatric inpatient facilities and by others in the same neighborhoods. Archive General of Psychiatry, May; 55(5): 393-401.

Trute, B; Tefft, B y Segall, a. (1989).- Social rejection of the mentally ill: a replication study of public attitude. Social Psychiatry and psychiatric epidemiology:24; 69-76.

Van Baar, A. (1990). Development of infants of drug dependent mothers. Child Psichology and Psychiatry. Sep; 31(6): 911-920.

Wahl, O.F. (1999). Mental health consumers experience of stigma. Schizophrenia Bulletin, 25, 467-478. 
